\title{
Role of systemic immune-inflammation index in patients treated with salvage radical prostatectomy
}

\author{
Pawel Rajwa ${ }^{1,2}\left(\right.$ D $\cdot$ Victor M. Schuettfort ${ }^{1,3} \cdot$ Fahad Quhal $^{1,4} \cdot$ Keiichiro Mori $^{1,5} \cdot$ Satoshi Katayama ${ }^{1,6} \cdot$ \\ Ekaterina Laukhtina $^{1,7} \cdot$ Benjamin Pradere ${ }^{1} \cdot$ Reza Sari Motlagh ${ }^{1,8} \cdot$ Hadi Mostafaei $^{1,9} \cdot$ Nico C. Grossmann $^{1,10}$. \\ Andreas Aulitzky ${ }^{1} \cdot$ Andrzej Paradysz $^{2} \cdot$ Pierre I. Karakiewicz $^{11} \cdot$ Harun Fajkovic $^{1,12} \cdot$ Kristin Zimmermann $^{13}$. \\ Axel Heidenreich ${ }^{14}$. Paolo Gontero ${ }^{15}$. Shahrokh F. Shariat ${ }^{1,7,12,16,17,18}$
}

Received: 1 February 2021 / Accepted: 26 April 2021 / Published online: 17 May 2021

(c) The Author(s) 2021

\begin{abstract}
Purpose To examine the predictive and prognostic value of preoperative Systemic Immune-inflammation Index (SII) in patients with radio-recurrent prostate cancer (PCa) treated with salvage radical prostatectomy (SRP).

Materials and methods This multicenter retrospective study included 214 patients with radio-recurrent $\mathrm{PCa}$, treated with SRP between 2007 and 2015. SII was measured preoperatively (neutrophils $\times$ platelets/lymphocytes) and the cohort was stratified using optimal cut-off. Uni- and multivariable logistic and Cox regression analyses were performed to evaluate the predictive and prognostic value of SII as a preoperative biomarker.

Results A total of 81 patients had high preoperative SII $(\geq 730)$. On multivariable logistic regression modeling, high SII was predictive for lymph node metastases (OR 3.32, 95\% CI 1.45-7.90, $p=0.005$ ), and non-organ confined disease (OR $2.55,95 \%$ CI 1.33-4.97, $p=0.005)$. In preoperative regression analysis, high preoperative SII was an independent prognostic factor for cancer-specific survival (CSS; HR 10.7, 95\% CI 1.12-103, $p=0.039)$ and overall survival (OS; HR 8.57, 95\% CI 2.70-27.2, $p<0.001$ ). Similarly, in postoperative multivariable models, SII was associated with worse CSS (HR 22.11, 95\% CI 1.23-398.12, $p=0.036$ ) and OS (HR 5.98, 95\% CI 1.67-21.44, $p=0.006$ ). Notably, the addition of SII to preoperative reference models improved the C-index for the prognosis of CSS (89.5 vs. 80.5) and OS (85.1 vs 77.1).

Conclusions In radio-recurrent PCa patients, high SII was associated with adverse pathological features at SRP and survival after SRP. Preoperative SII could help identify patients who might benefit from novel imaging modalities, multimodal therapy or a closer posttreatment surveillance.
\end{abstract}

Keywords SII $\cdot$ Biomarkers $\cdot$ Salvage radical prostatectomy $\cdot$ Prostate cancer $\cdot$ Survival

\section{Introduction}

Radiation therapy is an effective therapy for localized prostate cancer (PCa) with durable local control [1, 2]. After primary radiation, however, up to $50 \%$ of patients experience biochemical recurrence (BCR), which is associated with subsequent risk of metastasis and PCa-specific death [3-5]. While some of these patients develop distant recurrence, a large proportion would benefit from effective local salvage therapy [2]. One of them is salvage radical prostatectomy (SRP), which offers a possibility of cure, and is associated

Shahrokh F. Shariat

shahrokh.shariat@meduniwien.ac.at

Extended author information available on the last page of the article with 53\% 5-year recurrence-free survival (RFS) and over $70 \%$ 10-year cancer-specific survival (CSS) [2,6]. These favorable long-term outcomes after SRP comes at the cost of potentially significant adverse events, including incontinence, although improvement has been reported in recent years [2, 6-8]. This risk for adverse events could be acceptable if a cure or long-term remission can be achieved. The current prediction of outcomes based on clinicopathologic is, however, suboptimal. Considering the growing interest of salvage modalities in radio-recurrent PCa, there is an urgent need to improve risk stratification to guide treatment decision making with respect to radical, focal or systemic therapy $[2,5,6]$. The Systemic Immune-inflammation Index (SII) is a novel biomarker, which combines three immune cell counts into a simple formula: neutrophils $\times$ platelets/ 
lymphocytes [9]. Through the incorporation of single components of well-known prognostic biomarkers in urologic oncology, neutrophil-to-lymphocyte ratio (NLR) and platelet-to-lymphocyte ratio (PLR), the SII comprehensively depicts the cancer-related inflammatory burden $[9,10]$.

So far, the prognostic ability of the SII prognostic has been confirmed in the context of castration-resistant prostate cancer (CRPC) treated with systemic therapy but no data exist on SII in radio-recurrent PCa [9]. Therefore, we aimed to analyze the predictive and prognostic value of SII in a large cohort of radio-recurrent PCa patients who underwent SRP.

\section{Materials and methods}

We retrospectively reviewed data from five academic centers including patients with clinical non-metastatic radiationrecurrent PCa treated with SRP between 2007 and 2015. Local institutional review board approved this study (No. 1104011637). The database and follow-up have previously been described in detail [11, 12]. In general, patients were treated with primary radiation therapy, which included either brachytherapy or external beam radiation therapy (EBRT) or combination techniques (EBRT and brachytherapy, EBRT and intensity-modulated radiation therapy, or EBRT and three-dimensional conformal radiation therapy). BCR after RT was defined as PSA $\geq 2 \mathrm{ng} / \mathrm{ml}$ greater than the nadir (Phoenix criteria) [13]. Before SRP, all patients underwent confirmatory biopsy. No patient was diagnosed with imaging-detected metastases before SRP. All patients underwent SRP with pelvic lymph node dissection. PCa staging and grading were performed according to the 2007 American Joint Committee on Cancer Tumor Nodes Metastasis (TNM) staging system and 2006 Gleason grading consensus, respectively [14]. All prostate specimens were examined by dedicated genitourinary pathologists at all centers. Nonorgan confined disease was defined as $\mathrm{pT} \geq 3$ and/or $\mathrm{pN} \geq 1$; adverse pathology was defined as $\mathrm{pT} \geq 3$ and/or $\mathrm{pN} \geq 1$ and/ or $\mathrm{GS} \geq 8$ and/or positive surgical margins.

\section{Follow-up}

Patients generally underwent PSA testing and physical examination every 3 months within the first 2 years and every 6 months thereafter. We defined post-SRP BCR as $\mathrm{PSA} \geq 0.2 \mathrm{ng} / \mathrm{mL}$. No patients received adjuvant androgen deprivation therapy (ADT) before the diagnosis of BCR. Distant metastases were identified using radiologic imaging. The cause of death was retrieved from medical records and/ or death certificates. For PCa-specific death, only men with known recurrence after SRP, who had documented metastatic PCa, and who had PCa listed in the death certificate were considered to have died of PCa. We calculated followup from the date of RP to the date of death or last follow-up visit.

\section{Systemic immune-inflammation index (SII)}

SII data were retrieved from pre-SRP complete blood count and calculated as follows: neutrophils absolute count $\mathrm{x}$ platelets absolute count divided by lymphocytes absolute count. Preoperative SII cut-off point was determined by Receiver Operating Characteristics (ROC) curve analysis using the Youden index for cancer-specific survival (CSS). In summary, the Youden index provides the optimal cut-off from a continuous variable by showing the score that offers the best tradeoff between sensitivity and specificity. Using this score, the overall population was divided into two separate SII groups (low vs. high).

\section{Statistical analyses}

Associations between SII values and patients' clinicopathologic features were evaluated using the Wilcoxon rank-sum test for continuous variables and chi-square test of independence or Fisher's exact test for categorical variables, as appropriate. Univariable and multivariable logistic regression analyses tested the association of SII with adverse pathologic findings. The models' predictive accuracy was analyzed using receiver operating characteristics (ROC) curves and calculating the derived area under the curve (AUC). AUCs were statistically compared using DeLong's test. Kaplan-Meier estimates with log-rank testing were used to depict the association between preoperative SII and survival outcomes. Pre- and posttreatment univariable and multivariable Cox regression analyses analyzed the association of SII with BCR-free survival (BFS), metastasis-free survival (MFS), CSS, and overall survival (OS). $p$ value of $<0.05$ was considered as the threshold of statistical significance. All tests were two sided. Analyses were performed using R Version 4.0 (R Foundation for Statistical Computing, Vienna, Austria, 2020).

\section{Results}

Overall, 214 patients with radio-recurrent $\mathrm{PCa}$, who underwent SRP were included in our analyses. According to the optimal cut-off of $\geq 730,81$ patients were categorized to have high preoperative SII. Clinical and pathological features stratified by SII are presented in Table 1. Most of the patients had mild-to-severe concomitant diseases (83\% ASA 2-3), however, there were no significant differences between patients with low and high SII. There were significant differences between patients with low and high SII values in 
Table 1 Clinicopathologic features of 214 radio-recurrent patients treated with SRP for radio-recurrent $\mathrm{PCa}$

\begin{tabular}{|c|c|c|c|c|}
\hline \multirow[t]{2}{*}{ Characteristic } & \multirow{2}{*}{$\begin{array}{l}\text { Overall } \\
N=214\end{array}$} & \multicolumn{3}{|c|}{ Cohort stratified by SII } \\
\hline & & Low, $N=133$ & High, $N=81$ & $p$ value \\
\hline Age at SRP (IQR) & $69(64,72)$ & $69(64-73)$ & $69(64-72)$ & $>0.9$ \\
\hline ASA status $(\%)$ & & & & 0.11 \\
\hline 1 & $36(17)$ & $22(17)$ & $14(17)$ & \\
\hline 2 & $113(53)$ & $64(48)$ & $49(60)$ & \\
\hline 3 & $65(30)$ & $47(35)$ & $18(22)$ & \\
\hline BMI (IQR) & $24(24-27)$ & $24(24-27)$ & $24(24-27)$ & 0.4 \\
\hline Radiation therapy type & & & & 0.3 \\
\hline EBRT & $167(78 \%)$ & $101(76 \%)$ & $66(81 \%)$ & \\
\hline Brachytherapy & $39(18 \%)$ & $25(19 \%)$ & $14(17 \%)$ & \\
\hline EBRT + Brachytherapy & $8(3.7 \%)$ & $7(5.3 \%)$ & $1(1.2 \%)$ & \\
\hline PSA median (IQR) & $3.8(2.1-6.5)$ & $3.9(2.3-6.4)$ & $3.7(1.7-6.7)$ & 0.6 \\
\hline Pre-SRP biopsy GS (\%) & & & & 0.5 \\
\hline GS 6 & $48(22)$ & $32(24)$ & $16(20)$ & \\
\hline GS 7 & 104 (49) & $68(51)$ & $36(44)$ & \\
\hline GS 8 & $32(15)$ & $18(14)$ & $14(17)$ & \\
\hline GS 9 & $15(7.0)$ & $8(6.0)$ & $7(8.6)$ & \\
\hline GS 10 & $15(7.0)$ & $7(5.3)$ & $8(9.9)$ & \\
\hline Clinical staging (\%) & & & & 0.2 \\
\hline $\mathrm{cT} 1$ & $99(46)$ & $64(48)$ & $35(43)$ & \\
\hline cT2 & $84(39)$ & $54(41)$ & $30(37)$ & \\
\hline $\mathrm{cT} \geq 3$ & $30(14)$ & $14(11)$ & $16(20)$ & \\
\hline SRP GS (\%) & & & & 0.001 \\
\hline GS 6 & $14(6.5)$ & $11(8.3)$ & $3(3.7)$ & \\
\hline GS 7 & $114(53)$ & $83(62)$ & $31(38)$ & \\
\hline GS 8 & $43(20)$ & $20(15)$ & $23(28)$ & \\
\hline GS 9 & $30(14)$ & $14(11)$ & $16(20)$ & \\
\hline GS 10 & $13(6.1)$ & $5(3.8)$ & $8(9.9)$ & \\
\hline $\operatorname{PSM}(\%)$ & $43(20)$ & $20(15)$ & $23(28)$ & 0.029 \\
\hline pT3a (\%) & $92(43)$ & $49(37)$ & $43(53)$ & 0.029 \\
\hline pT3b $(\%)$ & $67(31)$ & $36(27)$ & $31(38)$ & 0.12 \\
\hline $\mathrm{pN} \geq 1(\%)$ & $40(19)$ & $15(11)$ & $25(31)$ & $<0.001$ \\
\hline OR (IQR) & $198(150-233)$ & $180(150-235)$ & $200(170-220)$ & 0.7 \\
\hline EBL (IQR) & $600(350-900)$ & $650(400-1000)$ & $600(350-885)$ & 0.3 \\
\hline Clavien-Dindo complication (\%) & & & & 0.079 \\
\hline 1 & $21(9.8)$ & $9(6.8)$ & $12(15)$ & \\
\hline 2 & $167(78)$ & $110(83)$ & $57(70)$ & \\
\hline 3 & $26(12)$ & $14(11)$ & $12(15)$ & \\
\hline
\end{tabular}

ASA American Society of Anesthesiologists; BMI body mass index; $E B L$ estimated blood loss; EBRT external beam radiation therapy; $G S$ Gleason score; $O R$ operating time; $P S A$ prostate-specific antigen; $P S M$ positive surgical margin; SRP salvage radical prostatectomy; SII Systemic Immune-inflammation Index Statistics presented: $n(\%)$; Median (IQR)

Statistical tests performed: Wilcoxon rank-sum test; chi-square test of independence; Fisher's exact test Significance bold values are $p<0.05$
SRP GS, positive surgical margins, extracapsular extension, and lymph node metastases at SRP. There were no differences between patients with low and high preoperative SII with regards to radiation therapy type, biopsy (pre-SRP) GS,
PSA level, age, and complications as assessed using Clavien-Dindo classification.

In univariable logistic regression analyses, high preoperative SII was associated with higher rates of $\mathrm{pT} \geq 3$ disease (odds ratio [OR] 1.94, HR 1.10-3.41, $p=0.021$ ), 
lymph node metastasis (OR 3.51, 95\% CI 1.72-7.18, $p=0.001$ ), non-organ confined disease (OR $2.50,95 \% \mathrm{CI}$ $1.40-4.45, p=0.002)$ and adverse pathology (OR 2.27, 95\% CI 1.27-4.07, $p=0.006$ ) (Supplementary Table I). In multivariable models that adjusted for the effect of the established clinicopathologic variables (Table 2), SII remained an independent predictive risk factor for lymph node metastasis (OR 3.32, 95\% CI 1.45-7.90, $p=0.005$ ), non-organ confined disease (OR 2.55, 95\% CI 1.33-4.97, $p=0.005$ ) and adverse pathology (OR 2.20, 95\% CI 1.15-4.33, $p=0.019$ ). The incorporation of preoperative SII into predictive reference models, comprising age, biopsy GS, preoperative PSA, and cT stage, did not significantly improve their accuracy with respect to the AUC for adverse pathological findings (Table 2).

The median follow-up was 25.3 (interquartile range [IQR], 15-28.5) months; 90 (42\%) patients experienced BCR, 23 (11\%) developed metastases, 7 (3.3\%) died from $\mathrm{PCa}$, and 18 (8.4\%) died from any cause. On Kaplan-Meier analyses, BFS, MFS, CSS, and OS were worse in patients with high preoperative SII compared to those with low SII (Fig. $1, p<0.05$ for all outcomes). On univariable Cox regression analyses, high SII was associated with BCR (HR $1.62,95 \%$ CI $1.07-2.46, p=0.024$ ), MFS (HR 3.09, 95\% $1.34-7.17, p=0.008$ ), CSS (HR 5.70, 95\% CI 1.09-29.80, $p=0.039$ ), and OS (HR 6.21, 95\% CI 2.30-16.76, $p<0.001$ ). In the preoperative multivariable regression models, high preoperative SII was an independent prognostic factor for CSS (HR 10.7, 95\% CI 1.12-103, $p=0.039$ ) and OS (HR 8.57 2.7-27.2, $p<0.001$ ), but not BCR (HR 1.39, 95\% CI $0.89-2.18, p=0.15$ ) or MFS (HR 2.09, 95\% CI 0.81-5.40, $p=0.129$ ) (Table 3). Similarly, in postoperative multivariable models, high SII was an independent prognostic factor for CSS (HR 22.11, 95\% CI 1.23-398.12, $p=0.036$ ) and OS (HR 5.98, 95\% CI 1.67-21.44, $p=0.006$ ) (Supplementary Table II). Incorporation of SII to reference preoperative models, comprising age, biopsy GS, PSA, and cT stage, resulted in the highest improvement of the discrimination ability for the prognosis of MFS (change of C-index of $5 \%$ ), CSS (change of C-index of 10\%), and OS (change of C-index of $9 \%$ ). In postoperative reference models, the inclusion of SII did not provide meaningful improvement to the C-index for any outcome (change of C-Index $\leq 4.2 \%$ for all outcomes).

\section{Discussion}

Local salvage therapy for radio-recurrent PCa is hampered by the accurate identification of localized versus systemic disease at the time of BCR after primary radiation with curative intent $[6,15]$. Current tolls fall short to their predictive accuracy to help guide in clinical decision making towards

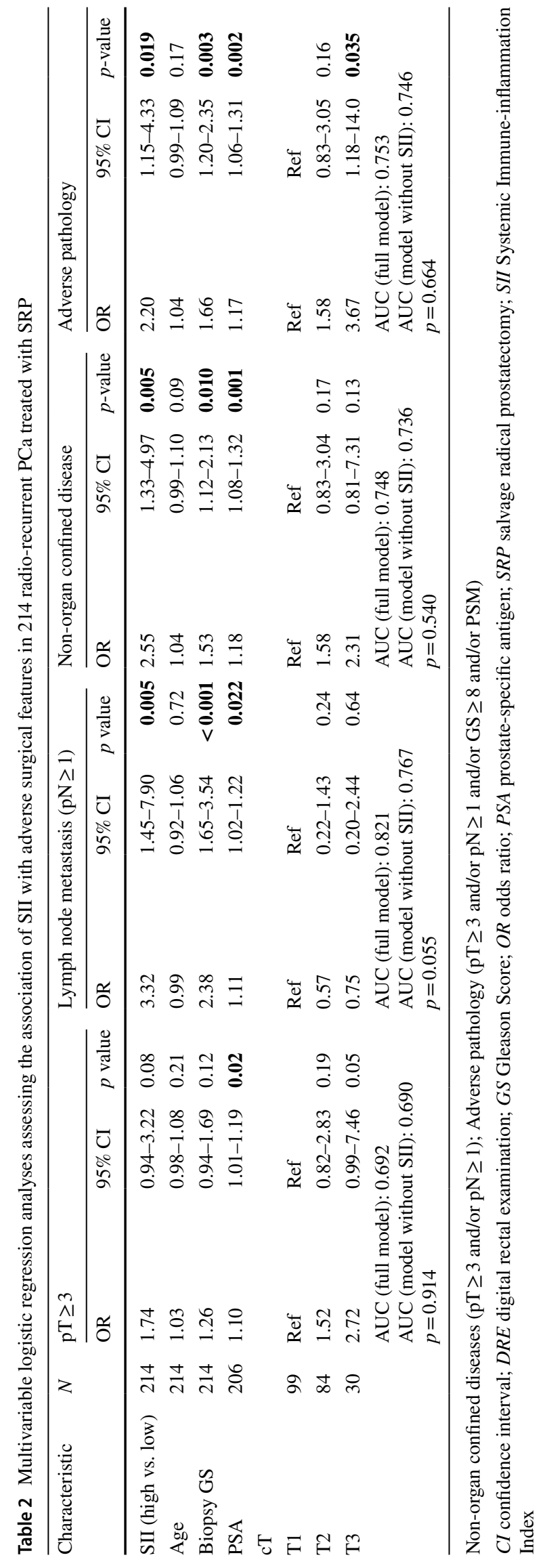



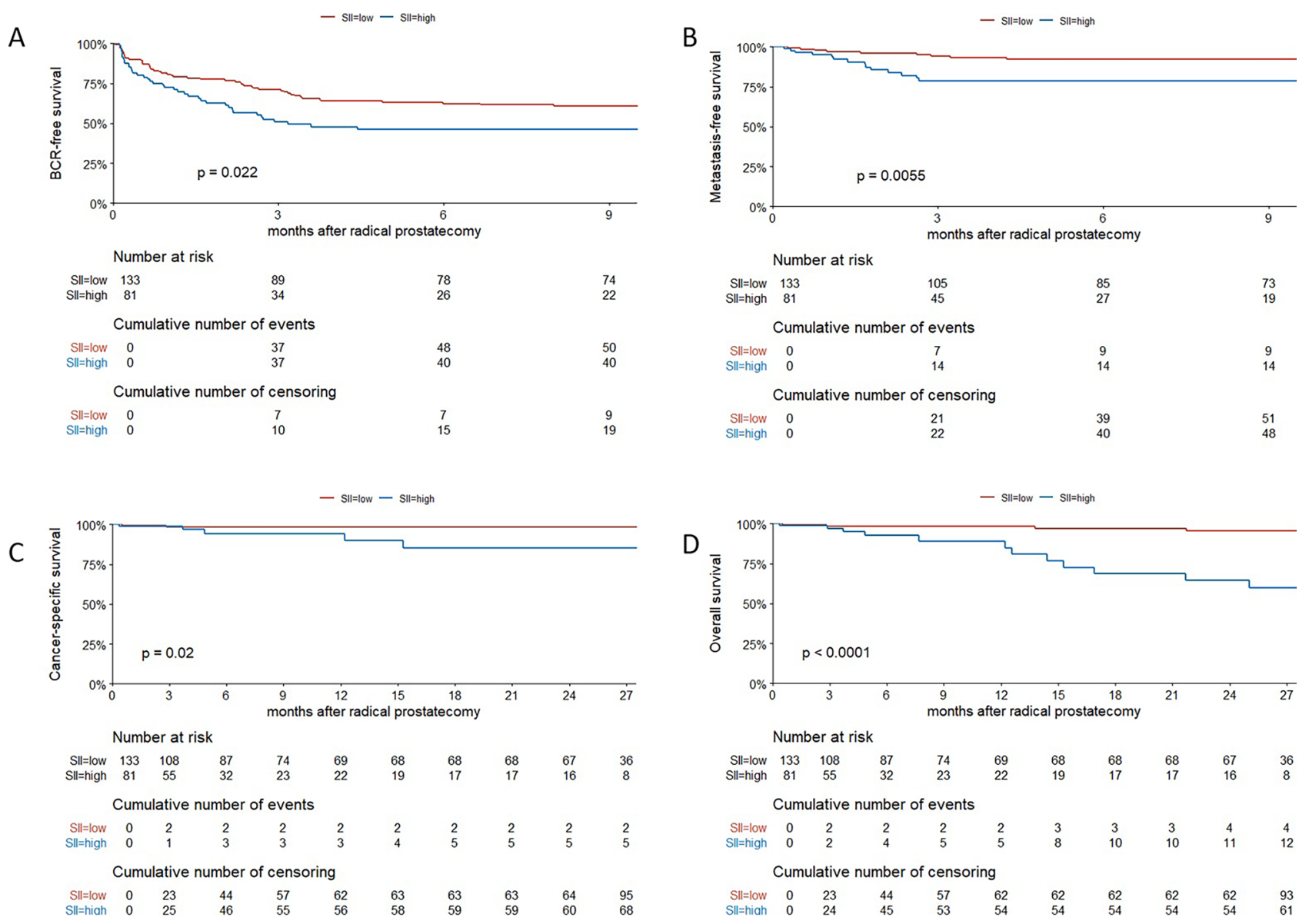

Fig. 1 Kaplan-Meier analysis stratified by SII levels for 214 patients treated with SRP for radio-recurrent PCa: a for BFS, b for MFS, $\mathbf{c}$ for CSS, d for OS

local salvage versus systemic therapy in this setting. Biomarkers that can capture the inherent biological aggressiveness of the tumor, as well as the host response, may help overcome the current staging and prognosis challenges [4, $16,17]$.

In our study, we found that preoperative SII predicts adverse pathologic findings at SRP and prognosticates survival outcomes in a large, multicenter cohort of patients treated with SRP for radio-recurrent PCa. Our results suggest that high SII can be used as a clinical guide to predict the probability of lymph node involvement, non-organ confined disease, and adverse pathology at SRP. In addition, high SII can be considered as a valuable prognostic factor for CSS and OS in radio-recurrent $\mathrm{PCa}$ patients treated with SRP. Notably, the incorporation of SII into the preoperative predictive models resulted in a clinically relevant increase of their predictive accuracy, especially with respect to CSS and OS.

No prior studies examined the role of SII in radio-recurrent PCa patients undergoing SRP. Our results indicate that patients with high SII had over three-time higher risk of being diagnosed with pathologically confirmed lymph node metastases and were over twice likely to harbor non-organ confined disease or adverse pathology. Also, the model including SII reached over $80 \%$ accuracy for the prediction of lymph node metastasis at SRP. These findings suggest that patients with higher SII levels should undergo more detailed imaging such as prostate-specific membrane antigen (PSMA) positron emission tomography (PET)/X-ray computed tomography (CT). Furthermore, patients with high SII could benefit from a more extensive approach at SRP, which includes extended lymph node dissection or re-irradiation or systemic therapy if surgery might be not technically feasible. Contrary, for patients with lower preoperative SII valuable option, may be focal therapy, which is associated with lower toxicity [2]. In PCa, the inflammatory burden has previously been linked to carcinogenesis and progression [18]. Furthermore, radiation therapy itself is known to trigger inflammatory (immune system) responses [19, 20]. All immune cells, which are components of SII, play a pivotal role in cancer response and cancer-related inflammation [10, 21-24]. Cancer cells facilitate pro-tumorigenic polarization 


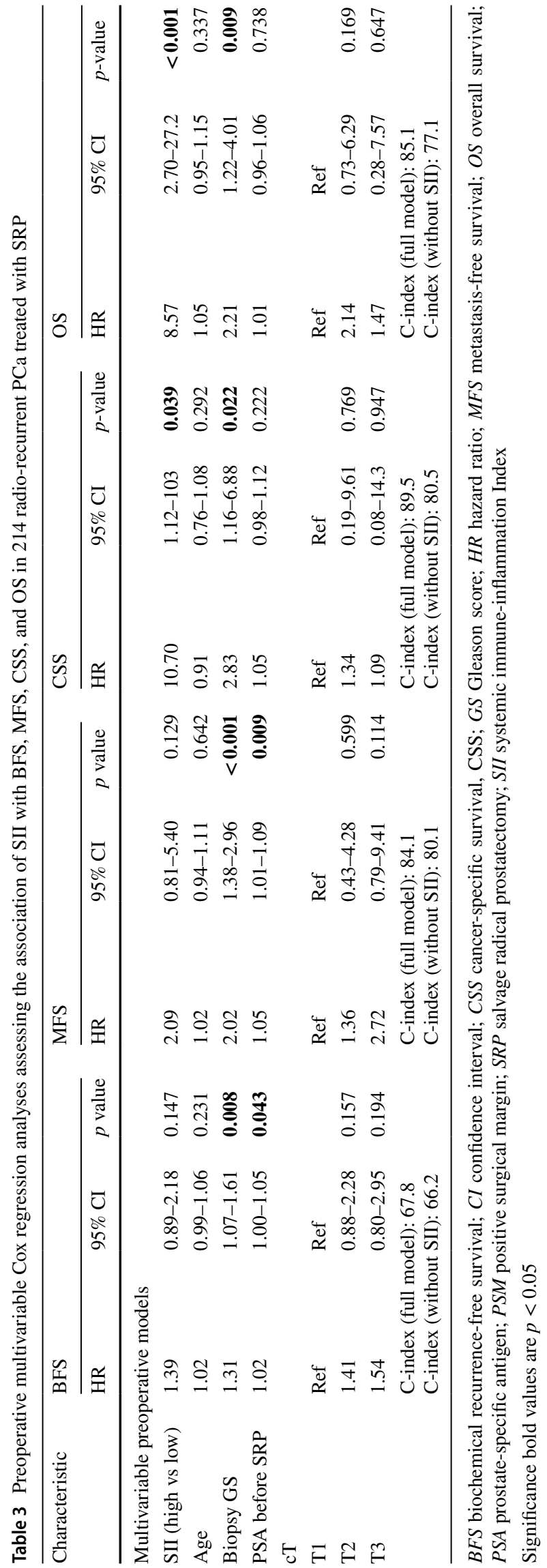

of neutrophils, which modulate the cancer microenvironment and other immune cells to promote tumor development [25]. Platelets have been suggested to contribute to tumor angiogenesis and metastasis [26]. A decreased number of lymphocytes may be a result of their cancer inhibition and is associated with impaired response to carcinogenesis [27, 28]. As a result, SII could serve as comprehensive biomarkers of inflammatory burden in radio-recurrent PCa.

In the case of primary radical prostatectomy, patients with adverse surgical features would undergo adjuvant radiation, but very little is known if (and how) these adverse features at SRP impact distant outcomes, and therefore, how to manage patients with locally advanced disease. Notably, in our cohort, high preoperative SII was a valuable, independent risk stratification tool for the two most important outcomes-CSS and OS. Despite the low number of these events, which are the likely cause for high hazard ratios and broad $95 \%$ confidence intervals, the association was robust, and SII enabled a clinically relevant increase of the accuracy of preoperative reference models. In this context, high preoperative SII has two important clinical implications. First, patients with high SII might be considered for other treatment modalities such as systemic therapy, as their clinical benefit of SRP is low. Second, if treated with SRP, these patients should undergo more scrutinous surveillance after SRP. In PCa, SII prognostic value was only evaluated in the context of CRPC patients treated with systemic therapy $[9,21,29]$. In a study of Man et al., high SII (> 535) was associated with OS in the multivariable model (HR 2.133, 95\% CI 1.163-3.913, $p=0.014$ ) [30]. Lolli et al. found that high SII ( $\geq 535$ ) was an independent prognostic factor for OS in CRPC patients treated with abiraterone (HR 2.08, 95\% CI 1.48-2.92, $p<0.01$ ) [29]. Furthermore, in another study, analyzing 104 patients with metastatic CRPC treated with sequential therapy, SII ( $\geq 200)$ prognosticated worse OS (HR 9.6, 95\% CI 4.7-19.5, $p<0.01$ ) and progressionfree survival (HR 17.4, 95\% CI 9.2-33.0, $p<0.01$ ) [31]. This is contradictory to the recent study of Stangl-Kremser et al. who did not find a significant association between SII $>200$ and overall survival in the CRPC cohort treated with docetaxel (HR 1.0, 95\% CI 0.9-1.0, $p=0.06$ ) [21]. The association between and SII was also reported in other solid tumors [23, 32]. For example, Hu et al. analyzed 646 non-metastatic renal cell carcinoma patients treated with nephrectomy and found high SII ( $>529)$ as an independent predictor of CSS (HR $=2.17,95 \%$ CI $1.33-3.55, p=0.002)$ and $\mathrm{OS}(\mathrm{HR}=2.26,95 \%$ CI $1.44-3.54, p<0.001)$ [32]. Also, in a recent meta-analysis of eight studies, Zhang et al. determined high SII as a prognostic factor for worse OS $(\mathrm{HR}=1.79,95 \% \mathrm{CI} 1.33-2.42, p<0.001)$ in breast cancer patients [23].

Our study has several limitations. This is a retrospective, multicenter study without central pathology examination and 
modest follow-up. Besides, patients were initially treated with various radiation therapy modalities and operated in multiple centers, and therefore surgical techniques and experiences could differ. Also, centers did not provide details on complications and concomitant diseases, but reported scores based on validated classifications (e.g. Clavien-Dindo and ASA). Furthermore, the SII level might have been biased by the presence of an autoimmune disease or chronic medical condition that can affect SII levels. Also, patients did not undergo PSMA PET-CT imaging for staging, which could result in the inclusion of patients with undetected metastases. Despite these flaws, we presented the first study, which comprehensively analyzed the role of SII in radio-recurrent PCa treated with SRP. Considering the paucity of available biomarkers in radio-recurrent PCa managed surgically, we believe our study provided substantial input to this field. Further studies with a prospective design are needed for validation of these results.

\section{Conclusions}

In radio-recurrent $\mathrm{PCa}$ patients, high SII was associated with adverse pathological features at SRP and survival after SRP. Preoperative SII could help identify patients who might benefit from novel imaging modalities, multimodal therapy or a closer posttreatment surveillance. Moreover, SII could improve the accuracy of currently utilized preoperative prognostic factors for CSS and OS. Further studies with a prospective design are needed for validation of these results.

Supplementary Information The online version contains supplementary material available at https://doi.org/10.1007/s00345-021-03715-4.

Acknowledgements VMS and EL are supported by the EUSP Scholarship of the European Association of Urology (EAU). PR is supported by the OMI/OSF Initiative: Promoting Brain Gain, Reducing Brain Drain in CEE.

Author contribution PR: protocol/project development, data analysis, data collection or management, manuscript writing/editing, VMS: data analysis, data collection or management, manuscript writing/editing, FH: data analysis, manuscript writing/editing, KM: data analysis, manuscript writing/editing, SK: manuscript writing/editing, EL: data analysis, manuscript writing/editing, BP: manuscript writing/ editing, RSM: manuscript writing/editing, HM: manuscript writing/ editing, NCG: manuscript writing/editing, AA: manuscript writing/ editing, AP: manuscript writing/editing, PIK: manuscript writing/editing, HF: manuscript writing/editing, KZ: manuscript writing/editing, AH: manuscript writing/editing, data collection or management, PG: manuscript writing/editing, data collection or management, SFS: protocol/project development, data collection or management, manuscript writing/editing.

Funding Open access funding provided by Medical University of Vienna.

\section{Declarations}

Conflict of interest The authors declare that they have no conflict of interest.

Ethics approval This article does not contain any studies with animals performed by any of the authors.

Informed consent Informed consent was obtained from all individual participants included in the study

Open Access This article is licensed under a Creative Commons Attribution 4.0 International License, which permits use, sharing, adaptation, distribution and reproduction in any medium or format, as long as you give appropriate credit to the original author(s) and the source, provide a link to the Creative Commons licence, and indicate if changes were made. The images or other third party material in this article are included in the article's Creative Commons licence, unless indicated otherwise in a credit line to the material. If material is not included in the article's Creative Commons licence and your intended use is not permitted by statutory regulation or exceeds the permitted use, you will need to obtain permission directly from the copyright holder. To view a copy of this licence, visit http://creativecommons.org/licenses/by/4.0/.

\section{References}

1. Mottet N, van den Bergh RCN, Briers E, Van den Broeck T, Cumberbatch MG, De Santis M, et al (2020) EAU-EANM-ESTROESUR-SIOG Guidelines on Prostate Cancer-2020 update. Part 1: screening, diagnosis, and local treatment with curative intent. Eur Urol. https://doi.org/10.1016/j.eururo.2020.09.042

2. Valle LF, Lehrer EJ, Markovic D, Elashoff D, Levin-Epstein R, Karnes RJ et al (2020) A systematic review and meta-analysis of local salvage therapies after radiotherapy for prostate cancer (MASTER). Eur Urol. https://doi.org/10.1016/j.eururo.2020.11. 010

3. Van den Broeck T, van den Bergh RCN, Arfi N, Gross T, Moris L, Briers E et al (2019) Prognostic value of biochemical recurrence following treatment with curative intent for prostate cancer: a systematic review. Eur Urol 75(6):967-987. https://doi.org/10. 1016/j.eururo.2018.10.011

4. Walz J, Gallina A, Perrotte P, Jeldres C, Trinh QD, Hutterer GC et al (2007) Clinicians are poor raters of life-expectancy before radical prostatectomy or definitive radiotherapy for localized prostate cancer. BJU Int 100(6):1254-1258. https://doi.org/10.1111/j. 1464-410X.2007.07130.x

5. Shariat SF, Kattan MW, Vickers AJ, Karakiewicz PI, Scardino PT (2009) Critical review of prostate cancer predictive tools. Future Oncol 5(10):1555-1584. https://doi.org/10.2217/fon.09.121

6. Chade DC, Shariat SF, Cronin AM, Savage CJ, Karnes RJ, Blute ML et al (2011) Salvage radical prostatectomy for radiation-recurrent prostate cancer: a multi-institutional collaboration. Eur Urol 60(2):205-210. https://doi.org/10.1016/j.eururo.2011.03.011

7. Gontero P, Marra G, Alessio P, Filippini C, Oderda M, Munoz $F$ et al (2019) Salvage radical prostatectomy for recurrent prostate cancer: morbidity and functional outcomes from a large multicenter series of open versus robotic approaches. J Urol 202(4):725-731. https://doi.org/10.1097/JU.0000000000000327

8. Heidenreich A, Richter S, Thuer D, Pfister D (2010) Prognostic parameters, complications, and oncologic and functional outcome of salvage radical prostatectomy for locally recurrent prostate 
cancer after 21st-century radiotherapy. Eur Urol 57(3):437-443. https://doi.org/10.1016/j.eururo.2009.02.041

9. Huang Y, Gao Y, Wu Y, Lin H (2020) Prognostic value of systemic immune-inflammation index in patients with urologic cancers: a meta-analysis. Cancer Cell Int 20:499. https://doi.org/10. 1186/s12935-020-01590-4

10. Mantovani A, Allavena P, Sica A, Balkwill F (2008) Cancerrelated inflammation. Nature 454(7203):436-444. https://doi.org/ 10.1038/nature07205

11. Vartolomei MD, D'Andrea D, Chade DC, Soria F, Kimura S, Foerster B et al (2019) Role of serum cholinesterase in patients treated with salvage radical prostatectomy. Urol Oncol 37(2):123-129. https://doi.org/10.1016/j.urolonc.2018.11.013

12. Quhal F, Pradere B, Sari Motlagh R, Mori K, Laukhtina E, Aydh A et al (2020) Prognostic value of preoperative albumin to globulin ratio in patients treated with salvage radical prostatectomy for radiation recurrent prostate cancer. Minerva Urol Nefrol. https:// doi.org/10.23736/S0393-2249.20.03938-7

13. Roach M 3rd, Hanks G, Thames H Jr, Schellhammer P, Shipley WU, Sokol GH et al (2006) Defining biochemical failure following radiotherapy with or without hormonal therapy in men with clinically localized prostate cancer: recommendations of the RTOG-ASTRO Phoenix Consensus Conference. Int J Radiat Oncol Biol Phys 65(4):965-974. https://doi.org/10.1016/j.ijrobp. 2006.04.029

14. Epstein JI, Allsbrook WC Jr, Amin MB, Egevad LL (2006) Update on the Gleason grading system for prostate cancer: results of an international consensus conference of urologic pathologists. Adv Anat Pathol 13(1):57-59. https://doi.org/10.1097/01.pap.00002 02017.78917.18

15. Shariat SF, Raptidis G, Masatoschi M, Bergamaschi F, Slawin KM (2005) Pilot study of radiofrequency interstitial tumor ablation (RITA) for the treatment of radio-recurrent prostate cancer. Prostate 65(3):260-267. https://doi.org/10.1002/pros.20242

16. Shariat SF, Khoddami SM, Saboorian H, Koeneman KS, Sagalowsky AI, Cadeddu JA et al (2004) Lymphovascular invasion is a pathological feature of biologically aggressive disease in patients treated with radical prostatectomy. J Urol 171(3):1122-1127. https://doi.org/10.1097/01.ju.0000113249.82533.28

17. Karam JA, Lotan Y, Roehrborn CG, Ashfaq R, Karakiewicz PI, Shariat SF (2007) Caveolin-1 overexpression is associated with aggressive prostate cancer recurrence. Prostate 67(6):614-622. https://doi.org/10.1002/pros.20557

18. Sfanos KS, De Marzo AM (2012) Prostate cancer and inflammation: the evidence. Histopathology 60(1):199-215. https://doi.org/ 10.1111/j.1365-2559.2011.04033.x

19. Jelonek K, Pietrowska M, Widlak P (2017) Systemic effects of ionizing radiation at the proteome and metabolome levels in the blood of cancer patients treated with radiotherapy: the influence of inflammation and radiation toxicity. Int J Radiat Biol 93(7):683696. https://doi.org/10.1080/09553002.2017.1304590

20. McKelvey KJ, Hudson AL, Back M, Eade T, Diakos CI (2018) Radiation, inflammation and the immune response in cancer. Mamm Genome 29(11-12):843-865. https://doi.org/10.1007/ s00335-018-9777-0

21. Stangl-Kremser J, Mari A, Suarez-Ibarrola R, D'Andrea D, Korn SM, Pones M, et al (2020) Development of a prognostic model for survival time prediction in castration-resistant prostate cancer patients. Urol Oncol 38(6):600e9-e15. https://doi.org/10.1016/j. urolonc.2019.11.005

22. Zhang JY, Ge P, Zhang PY, Zhao M, Ren L (2019) Role of neutrophil to lymphocyte ratio or platelet to lymphocyte ratio in prediction of bone metastasis of prostate cancer. Clin Lab 65(5). https:// doi.org/10.7754/Clin.Lab.2018.181040

23. Zhang Y, Sun Y, Zhang Q (2020) Prognostic value of the systemic immune-inflammation index in patients with breast cancer: a meta-analysis. Cancer Cell Int 20:224. https://doi.org/10.1186/ s12935-020-01308-6

24. Rajwa P, Zyczkowski M, Paradysz A, Slabon-Turska M, Suliga K, Bujak K et al (2020) Novel hematological biomarkers predict survival in renal cell carcinoma patients treated with nephrectomy. Arch Med Sci 16(5):1062-1071. https://doi.org/10.5114/ aoms.2017.70250

25. Coffelt SB, Wellenstein MD, de Visser KE (2016) Neutrophils in cancer: neutral no more. Nat Rev Cancer 16(7):431-446. https:// doi.org/10.1038/nrc.2016.52

26. Riedl J, Pabinger I, Ay C (2014) Platelets in cancer and thrombosis. Hamostaseologie 34(1):54-62. https://doi.org/10.5482/ HAMO-13-10-0054

27. Ray-Coquard I, Cropet C, Van Glabbeke M, Sebban C, Le Cesne A, Judson I et al (2009) Lymphopenia as a prognostic factor for overall survival in advanced carcinomas, sarcomas, and lymphomas. Cancer Res 69(13):5383-5391. https://doi.org/10.1158/ 0008-5472.CAN-08-3845

28. Kim R, Emi M, Tanabe K, Uchida Y, Toge T (2004) The role of Fas ligand and transforming growth factor beta in tumor progression: molecular mechanisms of immune privilege via Fas-mediated apoptosis and potential targets for cancer therapy. Cancer 100(11):2281-2291. https://doi.org/10.1002/cncr.20270

29. Lolli C, Caffo O, Scarpi E, Aieta M, Conteduca V, Maines F et al (2016) Systemic immune-inflammation index predicts the clinical outcome in patients with mCRPC treated with abiraterone. Front Pharmacol 7:376. https://doi.org/10.3389/fphar.2016.00376

30. Man YN, Chen YF (2019) Systemic immune-inflammation index, serum albumin, and fibrinogen impact prognosis in castrationresistant prostate cancer patients treated with first-line docetaxel. Int Urol Nephrol 51(12):2189-2199. https://doi.org/10.1007/ s11255-019-02265-4

31. Fan L, Wang R, Chi C, Cai W, Zhang Y, Qian H et al (2018) Systemic immune-inflammation index predicts the combined clinical outcome after sequential therapy with abiraterone and docetaxel for metastatic castration-resistant prostate cancer patients. Prostate 78(4):250-256. https://doi.org/10.1002/pros.23465

32. Hu X, Shao YX, Yang ZQ, Dou WC, Xiong SC, Li X (2020) Preoperative systemic immune-inflammation index predicts prognosis of patients with non-metastatic renal cell carcinoma: a propensity score-matched analysis. Cancer Cell Int 20:222. https://doi.org/ 10.1186/s12935-020-01320-w

Publisher's Note Springer Nature remains neutral with regard to jurisdictional claims in published maps and institutional affiliations. 


\section{Authors and Affiliations}

Pawel Rajwa ${ }^{1,2}(\mathbb{D}) \cdot$ Victor M. Schuettfort $^{1,3} \cdot$ Fahad Quhal $^{1,4} \cdot$ Keiichiro Mori $^{1,5} \cdot$ Satoshi Katayama ${ }^{1,6}$. Ekaterina Laukhtina $^{1,7}$. Benjamin Pradere ${ }^{1}$. Reza Sari Motlagh ${ }^{1,8} \cdot$ Hadi Mostafaei $^{1,9} \cdot$ Nico C. Grossmann $^{1,10}$. Andreas Aulitzky ${ }^{1}$. Andrzej Paradysz ${ }^{2}$. Pierre I. Karakiewicz ${ }^{11}$. Harun Fajkovic ${ }^{1,12}$ - Kristin Zimmermann ${ }^{13}$. Axel Heidenreich ${ }^{14}$. Paolo Gontero ${ }^{15}$. Shahrokh F. Shariat ${ }^{1,7,12,16,17,18}$

1 Department of Urology, Comprehensive Cancer Center, Medical University Vienna, Vienna General Hospital, Währinger Gürtel 18-20, 1090 Vienna, Austria

2 Department of Urology, Medical University of Silesia, Zabrze, Poland

3 Department of Urology, University Medical Center Hamburg-Eppendorf, Hamburg, Germany

4 Department of Urology, King Fahad Specialist Hospital, Dammam, Saudi Arabia

5 Department of Urology, The Jikei University School of Medicine, Tokyo, Japan

6 Department of Urology, Okayama University Graduate School of Medicine, Dentistry and Pharmaceutical Sciences, Okayama, Japan

7 Institute for Urology and Reproductive Health, Sechenov University, Moscow, Russia

8 Men's Health and Reproductive Health Research Center, Shahid Beheshti University of Medical Sciences, Tehran, Iran

$9 \quad$ Research Center for Evidence Based Medicine, Tabriz University of Medical Sciences, Tabriz, Iran
10 Department of Urology, University Hospital Zurich, Zurich, Switzerland

11 Cancer Prognostics and Health Outcomes Unit, University of Montreal Health Centre, Montreal, Canada

12 Karl Landsteiner Institute of Urology and Andrology, Vienna, Austria

13 Department of Urology, Federal Armed Services Hospital Koblenz, Koblenz, Germany

14 Department of Urology, University Hospital Cologne, Cologne, Germany

15 Division of Urology, Department of Surgical Sciences, San Giovanni Battista Hospital, University of Studies of Torino, Turin, Italy

16 Department of Urology, Weill Cornell Medical College, New York, NY, USA

17 Department of Urology, University of Texas Southwestern, Dallas, TX, USA

18 Department of Urology, Second Faculty of Medicine, Charles University, Prague, Czech Republic 